NBER WORKING PAPER SERIES

\title{
INDICATOR VARIABLES FOR OPTIMAL POLICY UNDER ASYMMETRIC INFORMATION
}

\author{
Lars E.O. Svensson \\ Michael Woodford
}

Working Paper 8255

http://www.nber.org/papers/w8255

\author{
NATIONAL BUREAU OF ECONOMIC RESEARCH \\ 1050 Massachusetts Avenue \\ Cambridge, MA 02138 \\ April 2001
}

We would like to thank Kosuke Aoki for helpful comments on an earlier draft. In addition, Lars Svensson thanks Princeton University for its hospitality during his visit 1999-2000, and Michael Woodford thanks the NSF for research support through a grant to the NBER. Remaining errors and expressed views are our own and not necessarily those of the NBER.

(C) 2001 by Lars E.O. Svensson and Michael Woodford. All rights reserved. Short sections of text, not to exceed two paragraphs, may be quoted without explicit permission provided that full credit, including $(\mathcal{C}$ notice, is given to the source. 
Indicator Variables for Optimal Policy under Asymmetric Information

Lars E.O. Svensson and Michael Woodford

NBER Working Paper No. 8255

April 2001

JEL No. E37, E47, E52, E58

\begin{abstract}
The optimal weights on indicators in models with partial information about the state of the economy and forward-looking variables are derived and interpreted, both for equilibria under discretion and under commitment. The private sector is assumed to have information about the state of the economy that the policymaker does not possess. Certainty-equivalence is shown to apply, in the sense that optimal policy reactions to optimally estimated states of the economy are independent of the degree of uncertainty. The usual separation principle does not hold, since the estimation of the state of the economy is not independent of optimization and is in general quite complex. We present a general characterization of optimal filtering and control in settings of this kind, and discuss an application of our methods to the problem of the optimal use of "real-time" macroeconomic data in the conduct of monetary policy.
\end{abstract}

Lars E.O. Svensson

Institute for International Economic Studies

Stockholm University

SE-106 91 Stockholm, Sweden

and NBER and CEPR

lars.svensson@iies.su.se
Michael Woodford

Department of Economics

Princeton University

Princeton, NJ 08544

and NBER

woodford@princeton.edu 


\section{Introduction}

Monetary policy is inevitably conducted under considerable uncertainty about the state of the economy and the nature of recent disturbances, and analyses of optimal policy that take no account of this are therefore of doubtful practical utility. However, in the case of purely backwardlooking models of the kind exclusively used by central banks prior to the 1990s, powerful general principles for efficient estimation of the state of the economy and for determining the optimal use to make of such estimates have been well-understood since at least the 1970s. In the case of a linear economic model, a quadratic loss function for the policymaker, and uncertainty only about the state of the economy (that is, the current values of certain additive terms in the economic model), a principle of certainty equivalence applies: the optimal policy is the same as if the state were fully observable, except that one responds to an efficient estimate of the state vector rather than to its actual value. Furthermore, a separation principle applies, according to which the determination of the optimal response coefficients to be applied to one's estimate of the state of the economy (the optimization problem) and the estimation of the current state of the economy (the estimation or signal-extraction problem) can be treated as separate problems. The optimal response coefficients are independent of the specification of the central bank's incomplete information; and the optimal weights to place on alternative indicators in estimating the state vector are independent of the central bank's objective function. ${ }^{1}$

This paper contributes to a program (initiated in Svensson and Woodford [12]) that seeks to determine the extent to which similar methods may be applied in the context of the sort of forward-looking models that are now widely used by central banks in policy simulations. Forward-looking models - that is, models in which the state of the economy is determined, among other factors, by expectations regarding the economy's future state — raise non-trivial complications not contemplated in the standard treatments of the 1970s. In [12], we consider a general class of linear-quadratic models, in which the private sector and the central bank are assumed to have the same partial information about the state of the economy. In this special case, we are able to establish that both certainty equivalence and a separation principle still obtain, when properly interpreted. We furthermore exhibit useful general formulas for computation of the optimal policy response coefficients and efficient estimates of the state of the economy in the context of a forward-looking (rational-expectations) model.

In the present paper, we instead consider the additional complications that arise in the

\footnotetext{
${ }^{1}$ Important early treatments include Chow [3], Kalchbrenner and Tinsley [5], and Leroy and Waud [6].
} 
case of a particular type of asymmetry between the information available to the central bank and that available to the private sector. The general problem of optimal policy in the case of asymmetric information appears to be quite difficult, so we here restrict our analysis to a particular, relatively simple case, that is nonetheless of considerable interest. ${ }^{2}$ This is the case in which the private sector has complete information about the current state of the economy, while the central bank observes only a particular set of indicators, which are also contaminated by "noise" that is unrelated to the fundamental determinants of the state variables that matter for its optimization problem.

One may or may not believe that central banks typically possess less information about the state of the economy than does the private sector. However, there is at least one important argument for the appeal of this assumption. This is that it is the only case in which it is intellectually coherent to assume a common information set for all members of the private sector, so that the model's equations can be expressed in terms of aggregative equations that refer to only a single "private sector information set," while at the same time these model equations are treated as structural, and hence invariant under the alternative policies that are considered in the central bank's optimization problem. It does not make sense that any state variables should matter for the determination of economically relevant quantities (that is, relevant to the central bank's objectives), if they are not known to anyone in the private sector. But if all private agents are to have a common information set, they must then have full information about the relevant state variables. ${ }^{3}$ It does not follow from this reasoning, of course, that it is more accurate to assume that all private agents have superior information to that of the central bank; it follows only that this case is one in which the complications resulting from partial information are especially tractable. The development of methods for characterizing optimal policy when different private agents have different information sets remains an important topic for further research. ${ }^{4}$

In this paper, we characterize both (Markov-perfect) equilibria in which the central bank optimizes under discretion and the optimal policy under commitment, giving particular attention

\footnotetext{
${ }^{2}$ Examples of recent applications of the kind of general analysis offered here to particular problems include Aoki [1], [2] and Dotsey and Hornstein [4]. The appendix to [4] independently derives some of the results presented in section 2 below for the case of discretionary policy, building upon the analysis in Svensson and Woodford [12] as we do. Here we also treat the more difficult case of optimal policy under commitment.

3 The kind of optimal policy problem treated in [12] is therefore not one for which rigorous microfoundations can be supplied. Relations such as the Lucas [7] aggregate supply equation, which appear to be of the form considered in that paper, are actually not fully structural; for they involve coefficients that are not invariant to changes in the policy regime, as Lucas stresses.

4 Important early studies that develop methods for characterizing rational expectations equilibria in which different private agents have different information sets include Townsend [14] Pearlman [9], and Sargent [10].
} 
to the optimal filtering problem of the central bank in each case. Our main results are parallel in both cases. We establish a certainty-equivalence principle once again: optimal instrument settings satisfy a feedback rule in which the coefficients (describing optimal central-bank responses to the bank's estimates of the state of the economy) are independent of the nature of the bank's partial information (though the estimates that the bank responds to depend upon this). On the other hand, we cannot obtain a separation principle of the sort obtained in the full information case, or in the case of symmetric partial information treated in [12]. The central bank's optimal estimates of the unobserved state variables (used as inputs to the feedback rules just described) are in general not independent of the objective function that it seeks to minimize. Furthermore, the complete equilibrium evolution of the state of the economy, given the path of the central bank's estimates, can no longer be derived independently of the specification of the bank's partial information and hence of the filter that it uses to derive its estimates. In this respect, asymmetric information results in complexities that do not arise in the case of symmetric partial information.

In section 2 , we set out the class of linear-quadratic models with which we are concerned, and then consider optimization under discretion. Section 3 presents corresponding results for the case of optimal policy with commitment. Finally, section 4 illustrates how our framework applies to the optimal policy problem considered by Aoki [1] and [2].

\section{Optimization under discretion}

We begin with an exposition of the general linear-quadratic framework that we assume throughout this paper, and then characterize optimizing policy under discretion.

\subsection{The framework}

We consider a linear model of an economy, in which a vector of state variables are determined by a system of structural equations intended to represent the conditions for rational-expectations equilibrium given optimizing private-sector behavior. We do not explicitly describe the optimization problems of private agents that underlie these equations, but we note that such optimization results in the presence of forward-looking terms (that is, conditional expectations) in the structural equations, and we take care to specify the common information set of all agents in the private sector, with respect to which these conditional expectations are defined. The optimization problem of the central bank (or more generally, the policymaker) is instead described 
explicitly; in this problem the structural equations resulting from private-sector optimization appear as constraints. We also take care to specify the information set of the central bank, which differs from that of the private sector.

The structural equations are given by a system of the form

$$
\left[\begin{array}{c}
X_{t+1} \\
\tilde{E} \mathrm{E}_{t} x_{t+1}
\end{array}\right]=A\left[\begin{array}{c}
X_{t} \\
x_{t}
\end{array}\right]+B i_{t}+\left[\begin{array}{c}
u_{t+1} \\
0
\end{array}\right],
$$

where $X_{t}$ is a vector of $n_{X}$ predetermined variables in period $t, x_{t}$ is a vector of $n_{x}$ forwardlooking variables, $i_{t}$ is (a vector of) the central bank's $n_{i}$ policy instrument(s), $u_{t}$ is a vector of $n_{X}$ iid shocks with mean zero and covariance matrix $\Sigma_{u u}$, and $A, B$ and $\tilde{E}$ are matrices of appropriate dimension. ${ }^{5}$ The $n_{x} \times n_{x}$ matrix $\tilde{E}$ (which should not be confused with the expectations operator $\mathrm{E}[\cdot]$ ) may be singular (this is a slight generalization of common formulations in which $\tilde{E}$ is the identity matrix).

We let $\mathrm{E}_{t} z_{\tau}$ denote the rational expectation (the best estimate) of any variable $z_{\tau}$ in period $\tau$, given private-sector information in period $t$. We will assume that the private-sector has full information, $I_{t}^{f}$, so $\mathrm{E}_{t} z_{\tau} \equiv \mathrm{E}\left[z_{\tau} \mid I_{t}^{f}\right]$. We let $z_{\tau \mid t}$ denote the rational expectation (the best estimate) of $z_{\tau}$ given the central-bank information in period $t$. We let $I_{t}$ denote central-bank information in period $t$, so $z_{\tau \mid t} \equiv \mathrm{E}\left[z_{\tau} \mid I_{t}\right]$. The information is further specified below.

We let $Y_{t}$ denote a vector of $n_{Y}$ target variables given by

$$
Y_{t}=C\left[\begin{array}{c}
X_{t} \\
x_{t}
\end{array}\right]+C_{i} i_{t}
$$

where $C$ and $C_{i}$ are matrices of appropriate dimension. We then let the quadratic form

$$
L_{t}=Y_{t}^{\prime} W Y_{t}
$$

be the central bank's period loss function, where $W$ is a positive-semidefinite weighting matrix.

Let the vector of $n_{Z}$ observable variables, $Z_{t}$, be given by

$$
Z_{t}=D\left[\begin{array}{c}
X_{t} \\
x_{t}
\end{array}\right]+v_{t}
$$

\footnotetext{
${ }^{5}$ In [12], we also allow the structural equations (2.1) that determine $X_{t}$ and $x_{t}$ to also include general linear terms involving the estimates $X_{t \mid t}$ and $x_{t \mid t}$ of the state of the economy based on partial information (of a kind specified below). However, the presence of such terms makes less sense when (as here) we assume that only the central bank has partial information. Equations (2.1) indicate how the state variables are determined by private-sector behavior given the central bank's instrument settings $i_{t}$, and the beliefs of the central bank (as opposed to those of the private sector) are not obviously relevant. Similar methods to those expounded here can be employed even in the presence of the additional terms, but at the cost of additional algebraic complexity.
} 
where $v_{t}$, the vector of noise, is iid with mean zero and covariance matrix $\Sigma_{v v}$. Central-bank information $I_{t}$ in period $t$ is given by

$$
I_{t}=\left\{Z_{\tau}, \tau \leq t ; A, B, C, C_{i}, D, \tilde{E}, W, \delta, \Sigma_{u u}, \Sigma_{v v}\right\}
$$

where $\delta(0<\delta<1)$ is a discount factor (to be introduced below). This incorporates the case when some or all of the predetermined and forward-looking variables are observable by the central bank. The full information $I_{t}^{f}$ in period $t$, the private-sector information set, is given by

$$
I_{t}^{f}=\left\{X_{\tau}, x_{\tau}, i_{\tau}, \tau \leq t ; A, B, C, C_{i}, D, \tilde{E}, W, \delta, \Sigma_{u u}, \Sigma_{v v}\right\}
$$

Thus, we here assume that the central bank has the same or less information than the private sector. The special case where both the central bank and the private sector have the same partial information is treated in Svensson and Woodford [12]. ${ }^{6}$

\subsection{Certainty equivalence}

In the present section, we assume that there is no commitment mechanism, so the central bank acts under discretion. Assume that central bank each period, conditional on the information $I_{t}$, minimizes the expected discounted current and future values of the intertemporal loss function,

$$
\mathrm{E}\left[\sum_{\tau=0}^{\infty} \delta^{\tau} L_{t+\tau} \mid I_{t}\right] .
$$

Svensson and Woodford [12, appendix A] show that certainty-equivalence applies for the central bank's optimization when the central bank and the private sector has the same information. The same proof goes through in the present case of asymmetric information. Thus, the equilibrium under discretion will be characterized by the instrument being a linear function of the current estimate of the predetermined variables,

$$
i_{t}=F X_{t \mid t}
$$

Furthermore, the estimate of the forward-looking variables will fulfill

$$
x_{t \mid t}=G X_{t \mid t},
$$

\footnotetext{
${ }^{6}$ Note that the predetermined and forward-looking variables can be interpreted as deviations from unconditional means and the target variables can be interpreted as deviations from constant target levels. More generally, constants, non-zero unconditional means and non-zero target levels can be incorporated by including unity among the predetermined variables, for instance, as the last element of $X_{t}$. The last row of the relevant matrices will then include the corresponding constants/means/target levels.
} 
where the matrix $G$ by [12, appendix A] fulfills

$$
G=\left(A_{22}-\tilde{E} G A_{12}\right)^{-1}\left[-A_{21}+\tilde{E} G A_{11}+\left(\tilde{E} G B_{1}-B_{2}\right) F\right]
$$

where the matrices $A$ and $B$ are decomposed according to $X_{t}$ and $x_{t}$,

$$
A \equiv\left[\begin{array}{ll}
A_{11} & A_{12} \\
A_{21} & A_{22}
\end{array}\right], B \equiv\left[\begin{array}{c}
B_{1} \\
B_{2}
\end{array}\right],
$$

and we assume that the matrix $A_{22}-\tilde{E} G A_{12}$ is invertible. The matrices $F$ and $G$ depend on $A, B, C, C_{i}, \tilde{E}, W$ and $\delta$, but (because of certainty equivalence) not on $D^{1}, D^{2}, \Sigma_{u u}$ and $\Sigma_{v v}$.

\subsection{Failure of the separation principle}

We can show that the forward-looking variables evolve according to a relation of the form

$$
x_{t}=G^{1} X_{t}+\left(G-G^{1}\right) X_{t \mid t},
$$

where the matrix $G^{1}$ remains to be determined. In the case of symmetric information, [12] shows that the matrix $G^{1}$ is given by

$$
G^{1}=-\left(A_{22}^{1}\right)^{-1} A_{21}^{1}
$$

and hence depends only on $A^{1}$ ( $A_{22}^{1}$ is assumed to be invertible). We will see that in the present case of asymmetric information, $G^{1}$ is determined in a more complex way. In particular, it is no longer generally independent of the specification of the central bank's partial information.

For a given $G^{1}$, it follows from $(2.1),(2.4),(2.8)$ and (2.11) that the dynamics for $X_{t}$ and $Z_{t}$ follow

$$
\begin{aligned}
X_{t+1} & =H X_{t}+J X_{t \mid t}+u_{t+1} \\
Z_{t} & =L X_{t}+M X_{t \mid t}+v_{t}
\end{aligned}
$$

where

$$
\begin{aligned}
H & \equiv A_{11}+A_{12} G^{1}, \\
J & \equiv B_{1} F+A_{12}\left(G-G^{1}\right), \\
L & \equiv D_{1}+D_{2} G^{1}, \\
M & \equiv D_{2}\left(G-G^{1}\right),
\end{aligned}
$$


and where $D \equiv\left[\begin{array}{ll}D_{1} & D_{2}\end{array}\right]$ is decomposed according to $X_{t}$ and $x_{t}$. (Note that the matrix $L$ in (2.17) should not be confused with the period loss function $L_{t}$ in (2.3).)

We note that, as in the symmetric-information case, the problem of estimating the predetermined variables has been transformed to a problem without forward-looking variables, with the transition equation (2.13) and the measurement equation (2.14); once again, the only nonstandard feature of this filtering problem is the circularity implied by the appearance of $X_{t \mid t}$ on the right-hand side of the measurement equation. Thus, as discussed in [12], with forward-looking variables among the observable variables, there is a simultaneity problem because the forwardlooking variables depend on the current estimate of the predetermined variables and the latter depend on the observables.

Temporarily ignoring the simultaneity problem, the optimal estimate of $X_{t+1}$ will be given by a Kalman filter updating equation,

$$
X_{t+1 \mid t+1}=X_{t+1 \mid t}+K\left(Z_{t+1}-L X_{t+1 \mid t}-M X_{t+1 \mid t+1}\right),
$$

where the Kalman gain matrix $K$ remains to be determined. The updating equation can, given (2.14), be written

$$
X_{t+1 \mid t+1}=X_{t+1 \mid t}+K\left[L\left(X_{t+1}-X_{t+1 \mid t}\right)+v_{t+1}\right] .
$$

The Kalman gain matrix is given by

$$
K=P L^{\prime}\left(L P L^{\prime}+\Sigma_{v v}\right)^{-1}
$$

where the matrix $P \equiv \operatorname{Cov}\left[X_{t+1}-X_{t+1 \mid t}\right]$ is the covariance matrix for the prediction errors $X_{t+1}-X_{t+1 \mid t}$ and satisfies

$$
P=H\left[P-P L^{\prime}\left(L P L^{\prime}+\Sigma_{v v}\right)^{-1} L P\right] H^{\prime}+\Sigma_{u u}
$$

Thus for given $G^{1}$ and hence given $H$ and $L, P$ can be solved from (2.22), either numerically or analytically, depending upon the complexity of the matrices $H, L$ and $\Sigma_{u u}$. Then $K$ is given by $(2.21)$.

The simultaneity in (2.19) means that the updating equation is not operational. Solving (2.19) for $X_{t+1 \mid t+1}$ eliminates the simultaneity and results in

$$
\begin{aligned}
X_{t+1 \mid t+1}= & (I+K M)^{-1}\left[(I-K L) X_{t+1 \mid t}+K Z_{t+1}\right] \\
& (I+K M)^{-1}\left[(I-K L)(H+J) X_{t \mid t}+K Z_{t+1}\right],
\end{aligned}
$$


where we have used the fact that by (2.13) the prediction equation will be

$$
X_{t+1 \mid t}=(H+J) X_{t \mid t}
$$

Equation (2.23) is an operational recursive updating equation, which avoids the simultaneity problem and uses the current observable variables to update the previous estimate $X_{t \mid t}$.

It only remains to determine $G^{1}$. Assuming a solution of the form (2.11), we must have

$$
\tilde{E} \mathrm{E}_{t} x_{t+1}=\tilde{E} G^{1} \mathrm{E}_{t} X_{t+1}+\tilde{E}\left(G-G^{1}\right) \mathrm{E}_{t} X_{t+1 \mid t+1} .
$$

We would like to express the right-hand side as a function of $X_{t}, x_{t}$ and $X_{t \mid t}$. We can then equate this with the second block of (2.1),

$$
\tilde{E} \mathrm{E}_{t} x_{t+1}=A_{21} X_{t}+A_{22} x_{t}+B_{2} F X_{t \mid t}
$$

(where we have used (2.9), solve for $x_{t}$ as a function of $X_{t}$ and $X_{t \mid t}$, and identify $G^{1}$.

We note that, by (2.13) and (2.20), we have

$$
\begin{aligned}
\mathrm{E}_{t} X_{t+1} & =H X_{t}+J X_{t \mid t} \\
\mathrm{E}_{t} X_{t+1 \mid t+1} & =X_{t+1 \mid t}+K L\left(\mathrm{E}_{t} X_{t+1}-X_{t+1 \mid t}\right) \\
& =(H+J) X_{t \mid t}+K L H\left(X_{t}-X_{t \mid t}\right) .
\end{aligned}
$$

Substituting this into (2.25), we obtain

$$
\begin{aligned}
\tilde{E} \mathrm{E}_{t} x_{t+1} & =\tilde{E} G^{1}\left(H X_{t}+J X_{t \mid t}\right)+\tilde{E}\left(G-G^{1}\right)\left[(H+J) X_{t \mid t}+K L H\left(X_{t}-X_{t \mid t}\right)\right] \\
& =\tilde{E}\left[G^{1}+\left(G-G^{1}\right) K L\right] H\left(X_{t}-X_{t \mid t}\right)+\tilde{E} G(H+J) X_{t \mid t} .
\end{aligned}
$$

Equating this with (2.26) and solving for $x_{t}$, we obtain a solution of the form (2.11), where $G^{1}$ must satisfy

$$
G^{1}=A_{22}^{-1}\left\{-A_{21}+\left[G^{1}+\left(G-G^{1}\right) K L\right] H\right\} .
$$

Thus, in contrast to (2.12) for the case where the private sector and central bank have the same information, the matrix $G^{1}$ now depends not only on $A$ but on $K L$ as well. Thus $G^{1}, H$, $L, K$ and $P$ are all simultaneously determined, by the equations (2.28), (2.15), (2.17), (2.21) and (2.22). In particular, since $K$ and $P$ depend on $\Sigma_{u u}$ and $\Sigma_{v v}$, so do the other matrices.

In the special case when $D_{2}=0$, that is, when none of the observable variables depend directly on any forward-looking variables, $L$ is by (2.17) independent of $G^{1}$ and hence of $K$. The 
other matrices are then still simultaneously determined. Recall that $F$ and $G$ only depend on $A, B, C, C_{i}, W$ and $\delta$, and not on $D, K$ or $L$, and hence not on $\Sigma_{u u}$ and $\Sigma_{v v}$.

In the case when the private sector has the same information as the central bank, the terms that are multiplied with the factor $G^{1}+\left(G-G^{1}\right) K L$ all vanish in $(2.27)$ (since $\mathrm{E}_{t} X_{t}-X_{t \mid t}=$ $\left.X_{t \mid t}-X_{t \mid t}=0\right)$. As a consequence, the terms multiplying that same factor in (2.28) vanish, and (2.28) reduces to $(2.12)$ as in $[12]$.

Thus, in the asymmetric-information case considered here, where the private-sector has full information and the central bank has partial information, certainty-equivalence still applies for the central bank's optimization problem, in the sense that the implied reaction function and response $F$ to its estimate of the predetermined variables is independent of the variance of the shocks, $\Sigma_{u u}$, and the information structure of the economy, $D^{1}, D^{2}$ and $\Sigma_{v v}$. However, the separation principle does not apply in the way that it does in the symmetric-information case. The estimation is now more complex and, in particular, it is not independent of the central bank's objective and its choice of $F$. (Note that $G^{1}$ by $(2.28)$ depends on $G$, which depends on $F$ and hence $C, C_{i}, W$ and $\delta$.)

\section{Optimal policy with commitment}

Again we assume the model (2.1), in which the private sector has full information, but now suppose instead that the central bank commits itself to an optimal plan for the indefinite future at information set $I_{0}$ in period 0 . As in the case of symmetric partial information treated in [12], the optimal commitment can be derived using a Lagrangian approach to what is essentially a planning problem in which the structural equations (2.1) are constraints. In the present informational assumptions, the Lagrangian takes the form

$$
\begin{aligned}
\mathcal{L}= & \mathrm{E}\left\{\sum_{t=0}^{\infty} \delta^{t} L_{t}-\sum_{t=0}^{\infty} \delta^{t} \varphi_{1, t+1}^{\prime}\left(X_{t+1}-A_{11} X_{t}-A_{12} x_{t}-B_{1} i_{t}\right)\right. \\
& \left.-\sum_{t=0}^{\infty} \delta^{t} \varphi_{2, t+1}^{\prime}\left(\tilde{E} \mathrm{E}_{t} x_{t+1}-A_{21} X_{t}-A_{22} x_{t}-B_{2} i_{t}\right)-\delta^{-1} \varphi_{10}^{\prime} X_{0} \mid I_{0}\right\},
\end{aligned}
$$

where in each period $t, \varphi_{1 t}$ and $\varphi_{2 t}$ are vectors of Lagrange multipliers conformable to $X_{t}$ and $x_{t}$ respectively. The multipliers $\varphi_{1, t+1}$ and $\varphi_{2, t+1}$ indicate the value of relaxing the constraints represented by the first and second rows of (2.1) respectively; the term $-u_{t+1}$ has been suppressed inside the first set of square brackets as it is irrelevant for the first-order conditions derived below. Because the second row of (2.1) represents a set of equilibrium conditions for the determination 
of $x_{t}$ as a function of information in $I_{t}^{f}$ (rather than for the determination of $x_{t+1}$ as a function of later information), the multipliers $\varphi_{2, t+1}$ are in fact measurable with respect to (that is, depend only on) the period $t$ information set $I_{t}^{f}$, whereas the multipliers $\varphi_{1, t+1}$ are measurable with respect to $I_{t+1}^{f}$. (The notation $\varphi_{2, t+1}$ rather than $\varphi_{2 t}$ is nonetheless convenient in writing expressions such as (3.1) below.) The final term on the right-hand side corresponds to the constraint imposed by the vector of initial conditions $X_{0}$.

\subsection{Certainty equivalence}

The Lagrangian can be written more compactly $^{7}$ in the form

$$
\mathcal{L}=\mathrm{E}\left\{\sum_{t=0}^{\infty} \delta^{t}\left[L_{t}+\varphi_{t+1}^{\prime}\left(A y_{t}+B i_{t}\right)-\delta^{-1} \varphi_{t}^{\prime} \tilde{I} y_{t}\right] \mid I_{0}\right\}
$$

where

$$
y_{t} \equiv\left[\begin{array}{c}
X_{t} \\
x_{t}
\end{array}\right], \quad \varphi_{t} \equiv\left[\begin{array}{c}
\varphi_{1 t} \\
\varphi_{2 t}
\end{array}\right], \quad \tilde{I} \equiv\left[\begin{array}{cc}
I & 0 \\
0 & \tilde{E}
\end{array}\right]
$$

We have added a term $-\delta^{-1} \varphi_{20}^{\prime} x_{0}$ to the terms inside the square brackets, for the sake of symmetry in notation, but now correspondingly stipulate the initial condition

$$
\varphi_{20}=0
$$

(Note that these Lagrange multipliers do not correspond to any actual constraint upon the planning problem.) This should be interpreted as an initial condition for each possible state in period zero consistent with the information set $I_{0}$, rather than a single initial condition for $I_{0}$.

Differentiation of (3.1) with respect to $y_{t}$ and $i_{t}$ then yields the first-order conditions

$$
\begin{gathered}
A^{\prime} \mathrm{E}_{t} \varphi_{t+1}+L_{y y} y_{t}+L_{y i} i_{t}-\delta^{-1} \tilde{I}^{\prime} \varphi_{t}=0, \\
B^{\prime} \varphi_{t+1 \mid t}+L_{i y} y_{t \mid t}+L_{i i} i_{t}=0,
\end{gathered}
$$

where the matrices $L_{j k}$ represent second partial derivatives of the period loss function. Note that equations (2.2) and (2.3) imply that $L_{t}$ is a quadratic function of $y_{t}$ and $i_{t}$,

$$
L_{t}=\left[\begin{array}{ll}
y_{t}^{\prime} & i_{t}
\end{array}\right]\left[\begin{array}{c}
C^{\prime} \\
C_{i}^{\prime}
\end{array}\right] W\left[\begin{array}{ll}
C & C_{i}
\end{array}\right]\left[\begin{array}{c}
y_{t} \\
i_{t}
\end{array}\right] \equiv \frac{1}{2}\left[\begin{array}{ll}
y_{t}^{\prime} & i_{t}
\end{array}\right]\left[\begin{array}{cc}
L_{y y} & L_{y i} \\
L_{i y} & L_{i i}
\end{array}\right]\left[\begin{array}{c}
y_{t} \\
i_{t}
\end{array}\right],
$$

\footnotetext{
${ }^{7}$ Here we use the fact that $\mathrm{E}\left[\varphi_{2, t+1} \mathrm{E}_{t} x_{t+1} \mid I_{0}\right]=\mathrm{E}\left[\mathrm{E}_{t} \varphi_{2, t+1} x_{t+1} \mid I_{0}\right]=\mathrm{E}\left[\varphi_{2, t+1} x_{t+1} \mid I_{0}\right]$, by the law of iterated expectations.
} 
so that the $L_{j k}$ are matrices of constant coefficients, that depend only upon the elements of the matrices $C, C_{i}$, and $W$.

Assuming that $L_{i i}$ is of full rank, ${ }^{8}$ we can solve (3.3) for $i_{t}$, obtaining

$$
i_{t}=-L_{i i}^{-1} L_{i y} y_{t \mid t}-L_{i i}^{-1} B^{\prime} \varphi_{t+1 \mid t}
$$

Substituting (3.5) into (2.1) and (3.2) to eliminate $i_{t}$, and taking the conditional expectation of both equations with respect to $I_{t}$, we then obtain a system of equations for the expected evolution of $y_{t}$ and $\varphi_{t}$, that can be written in the form

$$
\left[\begin{array}{cc}
0 & R^{\prime} \\
\tilde{I} & U
\end{array}\right]\left[\begin{array}{l}
y_{t+1 \mid t} \\
\varphi_{t+1 \mid t}
\end{array}\right]=\left[\begin{array}{cc}
V & \delta^{-1} \tilde{I}^{\prime} \\
R & 0
\end{array}\right]\left[\begin{array}{l}
y_{t \mid t} \\
\varphi_{t \mid t}
\end{array}\right],
$$

where

$$
\begin{aligned}
R & \equiv A-B L_{i i}^{-1} L_{i y}, \\
U & \equiv B L_{i i}^{-1} B^{\prime}, \\
V & \equiv-L_{y y}+L_{y i} L_{i i}^{-1} L_{i y} .
\end{aligned}
$$

Here it is worth noting that $U$ and $V$ are symmetric matrices.

Let us assume furthermore that the square matrix on the left-hand side of (3.6) is of full rank. Then we can invert this matrix, to obtain a system of the form

$$
\left[\begin{array}{l}
y_{t+1 \mid t} \\
\varphi_{t+1 \mid t}
\end{array}\right]=\mathcal{M}\left[\begin{array}{l}
y_{t \mid t} \\
\varphi_{t \mid t}
\end{array}\right]
$$

We then wish to consider solutions to (3.7) that are consistent with given initial values for $X_{t \mid t}$ and $\varphi_{2, t \mid t}$. We note that the number of variables in (3.7) is $2\left(n_{X}+n_{x}\right)$, where $n_{X}$ and $n_{x}$ is the dimension of $X_{t}$ and $x_{t}$, respectively, and that there are $n_{X}+n_{x}$ initial conditions $\left(X_{t \mid t}\right.$ and $\left.\varphi_{2, t \mid t}\right)$. We shall restrict our attention to bounded solutions, by which we mean solutions in which for any $t, y_{t+j \mid t}$ and $\varphi_{t+j \mid t}$ satisfy a uniform bound for all $j$. Such solutions necessarily satisfy the transversality condition for an optimal plan, and since our equations (2.1)-(2.4) will usually represent only a local approximation to the true structural equations and true loss function, unbounded solutions need not correspond at all closely to solutions to the true equations.

As usual (and ignoring non-generic cases), there is a unique bounded solution to (3.7) consistent with the initial conditions if the number of eigenvalues of $\mathcal{M}$ inside the unit circle (that

\footnotetext{
${ }^{8}$ The case in which $C_{i}=0$, with the consequence that $L_{i i}=0$, can be treated using similar methods, as shown in appendix $\mathrm{A}$.
} 
is, with modulus less than one) is exactly equal to the number of initial conditions, $n_{X}+n_{x}$. The eigenvalues $\lambda$ of $\mathcal{M}$ are the roots of the characteristic equation

$$
\operatorname{Det}\left[\begin{array}{cc}
V & \delta^{-1} \tilde{I}^{\prime}-\lambda R^{\prime} \\
R-\lambda \tilde{I} & -\lambda U
\end{array}\right]=0 .
$$

Multiplication of the right blocks of this matrix by $-\lambda^{-1}$, then multiplication of the lower blocks by $-\lambda^{-1} \delta^{-1}$, and finally transposition of the matrix does not change the sign of its determinant. Thus we may equivalently write

$$
\operatorname{Det}\left[\begin{array}{cc}
V & \delta^{-1} \tilde{I}^{\prime}-\lambda^{-1} \delta^{-1} R^{\prime} \\
R-\lambda^{-1} \delta^{-1} \tilde{I} & -\lambda^{-1} \delta^{-1} U
\end{array}\right]=0 .
$$

Comparison of this with (3.8) shows that if $\lambda$ is a root, $\lambda^{-1} \delta^{-1}$ must also be. It follows that $\mathcal{M}$ has as many eigenvalues with $|\lambda|>\frac{1}{\sqrt{\delta}}$ as with $|\lambda|<\frac{1}{\sqrt{\delta}}$. Thus, since $\frac{1}{\sqrt{\delta}}>1$, at most half of the eigenvalues (that is, at most $n_{X}+n_{x}$ ) are inside the unit circle (that is, with $|\lambda|<1$ ), so there is no possibility of multiple stationary solutions to (3.7). If $\delta$ is close to 1 (as will often be the case), there are likely to be exactly half inside the unit circle. We shall assume this condition from now on. ${ }^{9}$ Then (3.7) has a unique bounded solution in which $y_{t+j \mid t}$ and $\varphi_{t+j \mid t}$ can be expressed as linear functions of the initial conditions $X_{t \mid t}$ and $\varphi_{2, t \mid t}$, for arbitrary $j \geq 0$.

Substitution of this solution into (3.5) implies evolution of the instrument according to a relation of the form

$$
i_{t}=F X_{t \mid t}+\Phi \Xi_{t-1 \mid t}
$$

where $F$ and $\Phi$ are matrices of constant coefficients, and we now introduce the notation $\Xi_{t-1} \equiv$ $\varphi_{2 t}$ for the sub-vector of Lagrange multipliers that are predetermined state variables under our characterization of the optimal commitment, just as in the symmetric-information case treated in [12]. (We change the time subscript to emphasize that the elements of $\Xi_{t-1}$ are determined at date $t$-1.) Similarly, the conditional expectations of the forward-looking variables evolve according to a relation of the form

$$
x_{t \mid t}=G X_{t \mid t}+\Gamma \Xi_{t-1 \mid t}
$$

\footnotetext{
${ }^{9}$ In the case of an exact linear-quadratic model, as opposed to a mere local approximation to a nonlinear model, this condition is not necessary in order for us to identify the unique relevant solution to (3.7), as the unique solution that does not explode fast enough to violate the transversality condition. But in practice we are not likely to deal with models that we can regard as exact, and instead assume that the optimal plan happens to be stationary. In this case, the eigenvalue condition assumed in the text must hold, as there would otherwise be no stationary solution.
} 
while the conditional expectations of the Lagrange multipliers associated with the forwardlooking variables evolve according to

$$
\Xi_{t \mid t}=S X_{t \mid t}+\Sigma \Xi_{t-1 \mid t}
$$

where $G, \Gamma, S, \Sigma$ are further matrices of constant coefficients. Substitution of these solutions into (2.1) then implies that the conditional expectations of the predetermined variables evolve according to

$$
X_{t+1 \mid t}=(H+J) X_{t \mid t}+\Psi \Xi_{t-1 \mid t}
$$

where $H$ and $J$ are again defined as in (2.15)-(2.16), and

$$
\Psi \equiv A_{12} \Gamma+B_{1} \Phi
$$

These equations completely define the conditional expectations of both the state variables and the central bank's instrument settings at all future dates, as functions of the current estimates of the predetermined states $X_{t \mid t}$ and $\Xi_{t-1 \mid t}$. We note that all of the matrices $F, \Phi$, $G, \Gamma, S, \Sigma, H+J$, and $\Psi$ are exactly the same as in the case of the optimal plan with full information (and are independent of the matrices $D$ and $\Sigma_{v v}$ that define the partial information of the central bank); they are furthermore the same as in the case where there is no uncertainty at all (and independent of the matrix $\Sigma_{u u}$ defining the fundamental uncertainty). This is the sense in which the optimal commitment here continues to conform to the principle of certainty equivalence.

The system of equations (3.9)-(3.12) are also exactly the same as those that characterize the optimal commitment in the case of symmetric (though incomplete) information on the part of the central bank and the private sector, as shown in [12]. The only difference is that in the symmetric case, the Lagrange multipliers associated with the forward-looking variables satisfy $\Xi_{t-1 \mid t}=\Xi_{t-1 \mid t-1}=\Xi_{t-1}$, so that equations (3.9)-(3.12) can be written in terms of the multipliers themselves rather than their conditional expectations, while this is not generally true when the central bank has less information. (We show below that in general $\Xi_{t-1}$ depends on information not possessed by the central bank at date $t-1$.)

\subsection{Failure of the separation principle}

In the case of symmetric information, we can also take the second row of (2.1), subtract each term from its expectation conditional upon $I_{t}$, and obtain a set of $n_{x}$ linear relations between 
$x_{t}-x_{t \mid t}$ and $X_{t}-X_{t \mid t}$ that must hold at all times. (The derivation of these relations depends upon the fact that the $\mathrm{E}_{t} x_{t+1}$ term in the second row of (2.1) is instead replaced by $x_{t+1 \mid t}$ when the private sector has the same incomplete information as the central bank.) These relations can be solved for $x_{t}$, yielding (when (3.10) is used as well) a solution for $x_{t}$ as a linear function of $X_{t}, X_{t \mid t}$, and $\Xi_{t-1}$. Substituting this in turn into the first row of (2.1), one is able to solve for the complete dynamics of the state variables (and not merely their expectations conditional upon the central bank's information) and of the Lagrange multipliers given the evolution of the estimates $X_{t \mid t}$, with coefficients that are independent of the nature of the partial information. This is an aspect of the separation principle that can be shown to hold in the case of symmetric incomplete information, just as in the case of full information. However, that derivation (detailed in [13]) cannot be applied here, given that $\mathrm{E}_{t} x_{t+1}$ must be distinguished from $x_{t+1 \mid t}$.

However, the second row of (2.1) does imply that

$$
\tilde{I}\left(\mathrm{E}_{t} y_{t+1}-y_{t+1 \mid t}\right)=A \hat{y}_{t}
$$

where $\hat{y}_{t} \equiv y_{t}-y_{t \mid t}$. (Here we use the fact that $i_{t}$ must be measurable with respect to $I_{t}$.) Similarly, (3.2) implies that

$$
A^{\prime}\left(\mathrm{E}_{t} \varphi_{t+1}-\varphi_{t+1 \mid t}\right)=\delta^{-1} \tilde{I}^{\prime} \hat{\varphi}_{t}-L_{y y} \hat{y}_{t}
$$

where $\hat{\varphi}_{t} \equiv \varphi_{t}-\varphi_{t \mid t}$. Let us write the system (3.14)-(3.15) as

$$
\tilde{A}\left(\left[\begin{array}{c}
\mathrm{E}_{t} y_{t+1} \\
\mathrm{E}_{t} \varphi_{t+1}
\end{array}\right]-\left[\begin{array}{c}
y_{t+1 \mid t} \\
\varphi_{t+1 \mid t}
\end{array}\right]\right)=\tilde{B}\left[\begin{array}{c}
\hat{y}_{t} \\
\hat{\varphi}_{t}
\end{array}\right],
$$

where

$$
\tilde{A} \equiv\left[\begin{array}{cc}
\tilde{I} & 0 \\
0 & A^{\prime}
\end{array}\right], \quad \tilde{B} \equiv\left[\begin{array}{cc}
A & 0 \\
-L_{y y} & \delta^{-1} \tilde{I}^{\prime}
\end{array}\right]
$$

In order to solve (3.16) for the dynamics of $\hat{y}_{t}$ and $\hat{\varphi}_{t}$, we must specify how the central bank's conditional expectations are updated (for instance, how $y_{t+1 \mid t+1}$ relates to $y_{t+1 \mid t}$ ). Let us suppose that the Kalman filter for the bank's problem takes the form

$$
\left[\begin{array}{c}
y_{t+1 \mid t+1} \\
\varphi_{t+1 \mid t+1}
\end{array}\right]=\left[\begin{array}{l}
y_{t+1 \mid t} \\
\varphi_{t+1 \mid t}
\end{array}\right]+\tilde{K}\left[\bar{L}\left(\bar{X}_{t+1}-\bar{X}_{t+1 \mid t}\right)+v_{t+1}\right]
$$

where

$$
\bar{L} \equiv\left[\begin{array}{ll}
L & \Lambda^{1}
\end{array}\right] \text { and } \quad \bar{X}_{t} \equiv\left[\begin{array}{c}
X_{t} \\
\Xi_{t-1}
\end{array}\right]
$$


where $\tilde{K}$ and $\bar{L}$ are matrices that have yet to be identified. (We show below that the Kalman filter does indeed take this form, and identify these matrices.)

Equation (3.17) implies

$$
\left[\begin{array}{c}
\mathrm{E}_{t} y_{t+1 \mid t+1} \\
\mathrm{E}_{t} \varphi_{t+1 \mid t+1}
\end{array}\right]=\left[\begin{array}{c}
y_{t+1 \mid t} \\
\varphi_{t+1 \mid t}
\end{array}\right]+\tilde{K} \bar{L}\left(\mathrm{E}_{t} \bar{X}_{t+1}-\bar{X}_{t+1 \mid t}\right) .
$$

By adding $\left(\mathrm{E}_{t} y_{t+1}^{\prime}, \mathrm{E}_{t} \varphi_{t+1}^{\prime}\right)^{\prime}$ to both sides and rearranging, we can write the equation as

$$
\left[\begin{array}{c}
\mathrm{E}_{t} y_{t+1} \\
\mathrm{E}_{t} \varphi_{t+1}
\end{array}\right]-\left[\begin{array}{c}
y_{t+1 \mid t} \\
\varphi_{t+1 \mid t}
\end{array}\right]=\left[\begin{array}{c}
\mathrm{E}_{t} \hat{y}_{t+1} \\
\mathrm{E}_{t} \hat{\varphi}_{t+1}
\end{array}\right]+\tilde{K} \bar{L}\left(\mathrm{E}_{t} \bar{X}_{t+1}-\bar{X}_{t+1 \mid t}\right) .
$$

Let $\bar{I}$ be the submatrix of the identity matrix that selects the elements

$$
\bar{X}_{t} \equiv \bar{I}\left[\begin{array}{c}
y_{t} \\
\varphi_{t}
\end{array}\right]
$$

Then we can write (3.19) as

$$
\left[\begin{array}{c}
\mathrm{E}_{t} y_{t+1} \\
\mathrm{E}_{t} \varphi_{t+1}
\end{array}\right]-\left[\begin{array}{c}
y_{t+1 \mid t} \\
\varphi_{t+1 \mid t}
\end{array}\right]=(I-\tilde{K} \bar{L} \bar{I})^{-1}\left[\begin{array}{c}
\mathrm{E}_{t} \hat{y}_{t+1} \\
\mathrm{E}_{t} \hat{\varphi}_{t+1}
\end{array}\right]
$$

where we assume that $I-\tilde{K} \bar{L} \bar{I}$ is invertible. Substituting (3.20) into (3.16) results in

$$
\tilde{A}(I-\tilde{K} \bar{L} \bar{I})^{-1}\left[\begin{array}{c}
\mathrm{E}_{t} \hat{y}_{t+1} \\
\mathrm{E}_{t} \hat{\varphi}_{t+1}
\end{array}\right]=\tilde{B}\left[\begin{array}{c}
\hat{y}_{t} \\
\hat{\varphi}_{t}
\end{array}\right] .
$$

Again, we are interested in bounded solutions of (3.21) consistent with given initial values for $\hat{X}_{t}$ and $\hat{\Xi}_{t-1}$. We assume the appropriate configuration of eigenvalues of the system. There is then a unique bounded solution of the form

$$
\begin{aligned}
& \hat{x}_{t}=\bar{G}^{1}\left(\bar{X}_{t}-\bar{X}_{t \mid t}\right), \\
& \hat{\Xi}_{t}=\bar{S}^{1}\left(\bar{X}_{t}-\bar{X}_{t \mid t}\right),
\end{aligned}
$$

where as usual the matrices $\bar{G}^{1} \equiv\left[\begin{array}{ll}G^{1} & \Gamma^{1}\end{array}\right]$ and $\bar{S}^{1} \equiv\left[\begin{array}{ll}S^{1} & \Sigma^{1}\end{array}\right]$ can be derived from the eigenvectors of the system (3.21).

Combining (3.10) with (3.22), we find that

$$
x_{t}=\bar{G}^{1} \bar{X}_{t}+\left(\bar{G}-\bar{G}^{1}\right) \bar{X}_{t \mid t},
$$




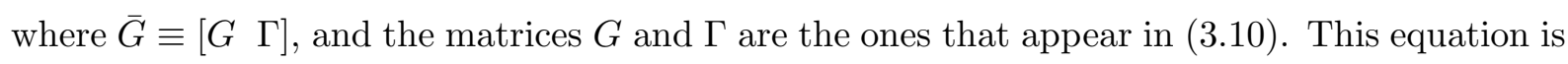
similar in form to the solution obtained in the case of symmetric partial information (discussed above), except that it includes separate terms in $\Xi_{t-1}$ and $\Xi_{t-1 \mid t}$ (which now differ), and that the matrix $G^{1}$ no longer takes the simple form (2.12). In particular, the matrix $G^{1}$ (and similarly $\Gamma^{1}$, which matters when $\left.\Xi_{t-1} \neq \Xi_{t-1 \mid t}\right)$ depends in general upon the elements of the Kalman gain matrix $\bar{K}$, and so is no longer independent of the nature of the central bank's partial information.

A complete solution for the dynamics of the state variables also now requires that we solve for the dynamics of the multipliers $\Xi_{t}$, which are no longer fully characterized by (3.11). Combining (3.11) and (3.22), we find that

$$
\Xi_{t}=\bar{S}^{1} \bar{X}_{t}+\left(\bar{S}-\bar{S}^{1}\right) \bar{X}_{t \mid t}
$$

where $\bar{S} \equiv\left[\begin{array}{ll}S & \Sigma\end{array}\right]$, and $S$ and $\Sigma$ are the matrices appearing in (3.11). It is the fact that $\bar{S}^{1}$ is generally non-zero in this derivation that implies that $\Xi_{t}$ is no longer measurable with respect to $I_{t}$. Unless all elements of $X_{t}$ are part of the central bank's information set at date $t+1, \Xi_{t}$ will generally not be measurable with respect to $I_{t+1}$, either.

It remains to determine the evolution of the estimates $\bar{X}_{t \mid t}$. We note that in the present case (unlike that of symmetric partial information) it is necessary to derive a Kalman filter for the evolution of the estimates $\Xi_{t-1 \mid t}$ as well as for the estimates $X_{t \mid t}$. Substitution of (3.9) and (3.24) into the first row of (2.1), combination with (3.25), and substitution into (2.4) leads to the transition equation for $\bar{X}_{t}$,

$$
\bar{X}_{t+1}=\bar{H} \bar{X}_{t}+\bar{J} \bar{X}_{t \mid t}+\bar{u}_{t+1}
$$

and to the measurement equation

$$
Z_{t}=\bar{L} \bar{X}_{t}+\bar{M} \bar{X}_{t \mid t}+v_{t}
$$

Here

$$
\bar{H} \equiv\left[\begin{array}{cc}
H & \Psi^{1} \\
S^{1} & \Sigma^{1}
\end{array}\right], \quad \bar{J} \equiv\left[\begin{array}{cc}
J & \Psi^{2} \\
S-S^{1} & \Sigma-\Sigma^{1}
\end{array}\right], \quad \bar{u}_{t} \equiv\left[\begin{array}{c}
u_{t} \\
0
\end{array}\right], \quad \bar{L} \equiv\left[\begin{array}{ll}
L & \Lambda^{1}
\end{array}\right], \quad \bar{M} \equiv\left[\begin{array}{ll}
M & \Lambda^{2}
\end{array}\right],
$$

$H, J, L, M$ are again defined as in (2.15)-(2.18), and

$$
\Psi^{1}=A_{12} \Gamma^{1}, \Psi^{2} \equiv A_{12}\left(\Gamma-\Gamma^{1}\right)+B_{1} \Phi, \Lambda^{1} \equiv D_{2} \Gamma^{1}, \Lambda^{2} \equiv D_{2}\left(\Gamma-\Gamma^{1}\right) .
$$


Note that $\Psi \equiv \Psi^{1}+\Psi^{2}$ is the same matrix as in (3.12), so that (3.26) is consistent with (though not implied by) equations (3.11) and (3.12) for the evolution of the conditional expectations.

The system of equations consisting of (3.26) then represents a system of transition equations with a form analogous to $(2.13)$, in which the state vector $X_{t}$ is replaced by the vector $\bar{X}_{t}$ that includes the elements of both $X_{t}$ and $\Xi_{t-1}$. Equation (3.27) is similarly a measurement equation with a form analogous to (2.14). It follows that the optimal estimates of $X_{t}$ and $\Xi_{t-1}$ will be given by a Kalman filter analogous to (2.20). We thus obtain updating equations for $X_{t}$ and $\Xi_{t-1}$ of the form indicated by the corresponding rows of (3.17),

$$
\bar{X}_{t+1 \mid t+1}=\bar{X}_{t+1 \mid t}+\bar{K}\left[\bar{L}\left(\bar{X}_{t+1}-\bar{X}_{t+1 \mid t}\right)+v_{t+1}\right],
$$

where $\bar{K} \equiv \bar{I} \tilde{K}$.

Using (3.26) to form the forecast $\bar{X}_{t+1 \mid t},(3.29)$ may be written

$$
\bar{X}_{t+1 \mid t+1}=(I-\bar{K} \bar{L})(\bar{H}+\bar{J}) \bar{X}_{t \mid t}+\bar{K}\left(\bar{L}_{X_{t+1}}+v_{t+1}\right) .
$$

For a given Kalman gain matrix $\bar{K}$, a complete system of equations for the evolution of the endogenous variables is then given by (3.24), (3.26) and (3.30), which apply in each period $t \geq 0$, starting from given initial values for $X_{0}$ and $X_{0 \mid 0}$, and from the initial values $\Xi_{-1}=\Xi_{-1 \mid 0}=0$.

It remains to determine the Kalman gain matrix in (3.29). Making use of the analogy between equations (3.26)-(3.29) and (2.13), (2.14) and (2.20), we directly find that the Kalman gain matrix is given by the equation

$$
\bar{K}=\bar{P} \bar{L}^{\prime}\left(\bar{L} \bar{P} \bar{L}^{\prime}+\Sigma_{v v}\right)^{-1}
$$

analogous to (2.21), where the matrix $\bar{P} \equiv \operatorname{Cov}\left[\bar{X}_{t}-\bar{X}_{t \mid t-1}\right]$ is the covariance matrix for the prediction errors $\bar{X}_{t}-\bar{X}_{t \mid t-1}$. The matrix $\bar{P}$ in turn satisfies the Riccati equation

$$
\bar{P}=\bar{H}\left[\bar{P}-\bar{P} \bar{L}^{\prime}\left(\bar{L} \bar{P} \bar{L}^{\prime}+\Sigma_{v v}\right)^{-1} \bar{L} \bar{P}\right] \bar{H}^{\prime}+\bar{\Sigma}_{u u}
$$

analogous to $(2.22)$, where

$$
\bar{\Sigma}_{u u} \equiv\left[\begin{array}{cc}
\Sigma_{u u} & 0 \\
0 & 0
\end{array}\right] .
$$

These equations are considerably more complicated to solve than in the case of symmetric partial information, treated in Svensson and Woodford [12]. First of all, the matrices $\bar{K}$ and $\bar{P}$ are larger (of dimension $\left(n_{X}+n_{x}\right) \times\left(n_{X}+n_{x}\right)$ rather than simply $\left.n_{X} \times n_{X}\right)$. But more 
importantly, the elements of the matrices $\bar{H}$ and $\bar{L}$ that appear in (3.28) do not depend solely upon the elements of $A$ and $D$, as in the case of symmetric information. For the matrix $G^{1}$ appearing in the definitions of $H$ and $L$ is no longer given by (2.12), but instead depends upon the matrices of the system (3.21) and hence on the Kalman gain matrix $\tilde{K}$ (though we show below that it only depends on $\bar{K}$ ). The same is true of the matrices $\Gamma^{1}, S^{1}$ and $\Sigma^{1}$ that enter into $\bar{H}$. Thus, one must solve a system of simultaneous equations for $\bar{P}, \bar{K}$, and the matrices that define the solution to (3.21); a separation principle no longer holds. This simultaneity is the same as we found in section 2, except that now the matrices involved are all of larger dimension.

The definitions of the matrices in (3.21) refer not only to $\bar{K}$ but also to the other elements of the Kalman gain matrix $\tilde{K}$. These, however, are easily expressed as functions of $\bar{K}$. Let us decompose $\tilde{K}$ with obvious notation,

$$
\tilde{K} \equiv\left[\begin{array}{c}
\tilde{K}_{X} \\
\tilde{K}_{x} \\
\tilde{K}_{\varphi 1} \\
\tilde{K}_{\varphi 2}
\end{array}\right]
$$

so that

$$
\bar{K} \equiv \bar{I} \tilde{K} \equiv\left[\begin{array}{c}
\tilde{K}_{X} \\
\tilde{K}_{\varphi 2}
\end{array}\right]
$$

Then by (3.10) the Kalman gain matrix for the forward-looking variables, $x_{t}$, is given by

$$
\tilde{K}_{x}=\bar{G} \bar{K}
$$

Similarly, the unique bounded solution to (3.7) allows us to write $\varphi_{1 t \mid t}$ as a matrix times $\bar{X}_{t \mid t}$, where the matrix is the same as in the case of full information (or certainty). This equation implies that the Kalman gain matrix for the prediction equation for $\varphi_{1 t}, \tilde{K}_{\varphi 1}$, will be the same matrix times $\bar{K}$. Thus the only simultaneity that must be dealt with is the dependence of the matrices $G^{1}, \Gamma^{1}, S^{1}$ and $\Sigma^{1}$ upon $\bar{K}$.

\section{Application: Optimal monetary policy with real-time data}

As an example of an application of our results, we sketch here the analysis of Aoki [1] and [2] of the way in which optimal monetary policy should make use of the preliminary estimates of current macroeconomic conditions that are available in real time. As Orphanides [8] in particular has stressed, policy must be made on the basis of preliminary estimates that are in fact revised 
substantially in subsequent months. Aoki models this by assuming that the available measures of current inflation and output are subject to measurement error, while the true values come to be known with a delay. The question that he considers is how the degree of uncertainty about the current state should affect the degree to which policy responds to the available real-time measures. Here we indicate how Aoki's analysis could be cast in terms of our framework.

Aoki assumes that output $y_{t}$ and inflation $\pi_{t}$ are determined by a model consisting of two structural equations,

$$
\begin{gathered}
y_{t}=E_{t} y_{t+1}-\sigma\left[i_{t}-E_{t} \pi_{t+1}-\rho_{t}\right], \\
\pi_{t}=\kappa\left(y_{t}-y_{t}^{n}\right)+\beta E_{t} \pi_{t+1},
\end{gathered}
$$

which can be interpreted as an intertemporal "IS equation" and "aggregate supply equation" respectively. Here the short-term nominal interest rate $i_{t}$ is assumed to be the central bank's policy instrument. The dependence of both relations upon expectations regarding future output and inflation introduces the forward-looking elements into the model's structural relation that complicates the central bank's optimal filtering and control problems. ${ }^{10}$ Note that the expectations in these relations are conditional upon the full information set of the private sector.

The exogenous disturbances $\rho_{t}$ and $y_{t}^{n}$ are assumed to evolve as first-order autoregressive processes,

$$
\begin{aligned}
\rho_{t} & =\eta \rho_{t-1}+e_{\rho t}, \\
y_{t}^{n} & =\nu y_{t-1}^{n}+e_{y t},
\end{aligned}
$$

where $0<\eta, \nu<1$ and the disturbances $e_{\rho t}, e_{y_{t}}$ are i.i.d. mean-zero normal random variables, with variance $s_{\rho}^{2}$ and $s_{y}^{2}$ respectively. The central bank is assumed to wish to minimize a discounted loss function (2.7), where the period loss function is of the form

$$
L_{t}=\pi_{t}^{2}+a\left(y-y_{t}^{n}\right)^{2}
$$

for a certain weight $a>0$. This is a standard specification of "flexible inflation targeting" (see Svensson [11]), and can also be justified as a utility-based welfare criterion given the individual decision problems underlying relations (4.1)-(4.2) (see Woodford [18]).

Because the "natural rate of output" around which one wishes to stabilize output according to (4.5) is exactly the shift factor in the aggregate supply relation (4.2), the aggregate supply

\footnotetext{
${ }^{10}$ See Woodford [17] for the derivation of these relations from the underlying decision problems of households and firms, and for further discussion of the model.
} 
relation itself implies no conflict between the goals of inflation and output-gap stabilization. It is possible in principle to completely stabilize both target variables, since $\pi_{t}=0$ and $y_{t}=y_{t}^{n}$ at all times is consistent with equation (4.2), and also with equation (4.1) as long as the nominal interest rate satisfies

$$
i_{t}=r_{t}^{n} \equiv \rho_{t}-\frac{1-\nu}{\sigma} y_{t}^{n} .
$$

That is, complete stabilization of both target variables is a possible equilibrium, as long as the interest rate $i_{t}$ perfectly tracks the exogenous variation in the Wicksellian "natural rate of interest" $r_{t}^{n}$ (discussed further in Woodford [17]). Under full information on the part of the central bank, this would represent optimal policy, as this equilibrium achieves the theoretical minimum value of the loss function (zero each period). ${ }^{11}$ However, the central bank's real-time information set may not allow it to estimate the current natural rate of interest with complete accuracy. In this case, complete stabilization is not feasible, and the central bank faces a tradeoff between the two goals of greater inflation or greater output-gap stabilization.

Aoki assumes that the central bank's information set when setting $i_{t}$ consists of complete information about all state variables known to the private sector at date $t-1$, plus noisy preliminary estimates of current inflation and output. These preliminary estimates are assumed to be given by

$$
\begin{aligned}
\pi_{t}^{o} & =\pi_{t}+\varepsilon_{\pi t}, \\
y_{t}^{o} & =y_{t}+\varepsilon_{y t},
\end{aligned}
$$

where the measurement-error terms $\varepsilon_{\pi t}$ and $\varepsilon_{y t}$ are i.i.d. mean-zero normal random variables, with variance $\sigma_{\pi}^{2}$ and $\sigma_{y}^{2}$ respectively. These errors in the central bank's preliminary estimates are assumed to be distributed independently of the "fundamental" disturbances $e_{\rho t}$ and $e_{y t}$.

Aoki's model falls within the general framework set out in the previous sections. The structural equations (4.1)-(4.2), together with laws of motion (4.3)-(4.4) for the disturbances, comprise a model of the form (2.1). Here the predetermined/exogenous state variables consist of a 2-vector of exogenous disturbances $X_{t}^{*}$ and the non-predetermined endogenous variables consist

\footnotetext{
11 That is, an optimal policy would bring about an equilibrium in which the nominal interest rate would vary in this way. This does not necessarily mean that the optimal policy is for the central bank to set its instrument according to the function (4.6) of the exogenous disturbances; for while such a policy rule would be consistent with the optimal equilibrium, it would also allow an extremely large class of other rational-expectations equilibria as well, which equilibria are less desirable. Other rules, that specify feedback from inflation and outputgap outcomes as under the "Taylor rule", are equally consistent with the optimal equilibrium and can render equilibrium determinate as well (see Woodford [17]). There is no uniquely optimal rule of this form, since rules that differ in the way that they specify out-of-equilibrium behavior may imply the same set of equilibria.
} 
of a 2-vector $x_{t}$, where

$$
X_{t}^{*} \equiv\left[\begin{array}{c}
\rho_{t} \\
y_{t}^{n}
\end{array}\right], \quad x_{t} \equiv\left[\begin{array}{c}
\pi_{t} \\
y_{t}
\end{array}\right]
$$

and it is possible to write equations (4.1)-(4.2) in a form such that $\tilde{E}=I$. While it would be possible to write the model's equations in the form (2.1) with $X_{t}=X_{t}^{*}$, this notation would not allow us to express the central bank's period $t$ observables as a function of $X_{t}, x_{t}$ and measurement error alone, as assumed in (2.4). In order to directly apply our above expressions for the optimal filtering problem, it is necessary to augment the state vector to include lagged values of the fundamental disturbances, so that

$$
X_{t}=\left[\begin{array}{c}
X_{t}^{*} \\
X_{t-1}^{*}
\end{array}\right]
$$

The system (2.1) then becomes a system of six difference equations, including two identities.

Aoki's loss function (4.5) also falls under our framework. This is a loss function of the form (2.3), where

$$
C=\left[\begin{array}{cccccc}
0 & 0 & 0 & 0 & 1 & 0 \\
0 & -1 & 0 & 0 & 0 & 1
\end{array}\right], \quad C_{i}=0, \quad W=\left[\begin{array}{cc}
1 & 0 \\
0 & a
\end{array}\right]
$$

Because $C_{i}=0$, we must apply the approach described in appendix A rather than the one followed in the text, but we are still able to derive a system of first-order conditions for optimal policy of the form (3.7).

Finally, the assumed information structure falls under the framework set out above, if we let

$$
Z_{t} \equiv\left[\begin{array}{c}
Z_{t}^{*} \\
X_{t-1}^{*}
\end{array}\right]=\left[\begin{array}{ccc}
I & 0 & 0 \\
0 & I & 0
\end{array}\right]\left[\begin{array}{c}
X_{t}^{*} \\
X_{t-1}^{*} \\
x_{t}
\end{array}\right]+\left[\begin{array}{c}
\varepsilon_{t} \\
0
\end{array}\right],
$$

where $Z_{t}^{*}$ and $\varepsilon_{t}$ are the 2 -vectors of the observations, $\left[\pi_{t}^{o} y_{t}^{o}\right]^{\prime}$, and measurement errors, $\left[\varepsilon_{\pi t} \varepsilon_{y t}\right]^{\prime}$, respectively. Under this specification, the central bank's information set at date $t$ includes the entire history $\left\{X_{t-j}^{*}\right\}$ for $j \geq 1$, and so a complete description of the state of the world as of date $t-1$, but only the information about date $t$ fundamentals that is contained in the noisy measurements of current inflation and output (the two elements of $Z_{t}^{*}$ ). Aoki assumes, as we do, complete information on the part of the private sector.

Our results in sections 2 and 3 can then be directly applied. Discretionary optimization by the central bank (treated by Aoki in [1]) results in the interest rate satisfying a relation of the 
form (2.8). Furthermore, the matrix $F$ in this relation is the same as under full information. This allows us to write the relation in the simpler form

$$
i_{t}=f^{\prime} X_{t \mid t}^{*}
$$

since the state variables $X_{t-1}^{*}$ may be omitted from the vector $X_{t}$ in the case of full information. Furthermore, the corresponding relation in the full-information case is given by (4.6), allowing us to identify the elements of the vector $f^{\prime}$. Optimal policy under discretion is thus characterized by the relation

$$
i_{t}=r_{t \mid t}^{n}
$$

A Kalman filter can then be used to describe the evolution of the central bank's optimal estimate of the current natural rate of interest.

Under an optimal commitment (treated by Aoki in [2]), instead, the interest rate satisfies a more complex relation of the form

$$
i_{t}=f^{\prime} X_{t \mid t}^{*}+\phi^{\prime} \Xi_{t-1}
$$

(This similarly follows from (3.9), given that the elements $X_{t-1}^{*}$ of the state vector $X_{t}$ are irrelevant in the case of full information, and that $\Xi_{t-1 \mid t}=\Xi_{t-1}$ under Aoki's information structure.) Once again, the vectors $f^{\prime}$ and $\phi^{\prime}$ are the same as in the case of full information. Since the optimal equilibrium under full information satisfies (4.6), ${ }^{12}$ the vector $f^{\prime}$ is the same as in the case of discretionary optimization, and (4.10) may equivalently be written

$$
i_{t}=r_{t \mid t}^{n}+\phi^{\prime} \Xi_{t-1}
$$

Note that it would not be correct to argue on the basis of certainty equivalence that since $i_{t}=r_{t}^{n}$ is the optimal commitment under full information, the optimal rule with incomplete information will satisfy $i_{t}=r_{t \mid t}^{n}$. The reason that the optimal commitment (4.6) can be written without any feedback from a Lagrange multiplier in the case of full information is not because $\phi^{\prime}=0$ for this model, but rather because $\Xi_{t-1}=0$ at all times. This is because under full information, complete stabilization of the target variables is feasible, and so there is no gain from relaxing the constraints imposed by the model's structural equations. But one can show (by computing the relevant eigenvectors of the matrix $\mathcal{M}$ in (3.7)) that $\phi^{\prime} \neq 0$, so that the second term on the right-hand side of (4.11) cannot be omitted. Nor is it true that $\Xi_{t-1}$ is

\footnotetext{
${ }^{12}$ Note that in the case of full information, (4.6) describes the result of central-bank optimization under either discretion or commitment - the optimal commitment happens in this case to be time-consistent.
} 
zero most of the time, in the case of imperfect information on the part of the central bank. This is because when information is imperfect, it will generally not be possible for the central bank to adjust its interest-rate instrument (which must depend only upon the central bank's information) so as to perfectly track variations in the natural rate of interest, and so perfect stabilization will be unattainable. In this case, there will generally be non-zero state-contingent Lagrange multipliers associated with perturbations of the constraints implied by the structural equations (4.1)-(4.2).

This does not contradict our certainty-equivalence results above, because these do not imply that the law of motion for the Lagrange multipliers must be the same as in the case of full information. It is true that (3.11) must hold, with the same matrices $S$ and $\Sigma$ as in the case of full information. In the case of full information, we know that $S=0$, since $\Xi_{t}$ will be zero regardless of the realization of the exogenous disturbances $X_{t}$. Thus (3.11) reduces in Aoki's model to

$$
\Xi_{t \mid t}=\Sigma \Xi_{t-1 \mid t}=\Sigma \Xi_{t-1}
$$

for a certain matrix $\Sigma$. However, $\Sigma$ is not a zero matrix. In the case of full information, the corresponding law of motion

$$
\Xi_{t}=\Sigma \Xi_{t-1}
$$

implies that $\Xi_{t}=0$ for all $t$ as a result of the initial condition $\Xi_{-1}=0$, despite the fact that $\Sigma \neq 0$. But with incomplete information, the initial condition $\Xi_{-1}=0$ no longer implies that $\Xi_{t}=0$ as well at all later dates. Instead, it implies only that $\Xi_{t \mid 0}=0$ for all $t \geq 0$. The actual value of the Lagrange multiplier will be given by the law of motion

$$
\Xi_{t}=S^{1}\left[X_{t}-X_{t \mid t}\right]+\Sigma \Xi_{t-1}
$$

where $S^{1} \neq 0$. Thus (4.11) makes $i_{t}$ a function not only of the central bank's period- $t$ estimate of the current state, but also of what it has learned by period $t$ about its past errors in estimation of the economy's state.

Specifically, Aoki [2] shows that the optimal commitment involves making interest rates higher than the current estimate of the natural rate if the bank now knows that it underestimated the natural rate in the past. The reason is that the private sector is aware of the bank's misperception, and if it understands that the bank will later correct its error, then even when the current short rate is below the natural rate, the private sector's expectation of higher future short rates will dampen the high demand (and hence inflationary pressure) that would otherwise 
result. Of course, the cost of fulfilling such a commitment later is a short-term interest rate that deviates to a greater extent from the natural rate at that time; but some use of this mechanism can nonetheless improve the bank's overall stabilization objectives. Optimal policy is thus not purely forward-looking, in the sense discussed in Woodford [16]. Indeed, the response of the bank's interest-rate instrument to fluctuations in the natural rate of interest will be more inertial than is the natural rate itself, just as in the analysis of Woodford [15]. In the case of a positive innovation in the natural rate, the average immediate increase in the nominal interest rate will be smaller, because of the bank's inability to identify the increase immediately given its imperfect observation of current conditions. At the same time, the increase in interest rates will last longer, because in later periods the central bank will respond to its initial underestimate of the natural rate by keeping interest rates higher than the natural rate at those later times. Interestingly, Aoki obtains these results in the case of asymmetric information without any need for the assumption of an interest-rate stabilization objective in the central bank's loss function, relied upon in the full-information analysis of Woodford [15]. 


\section{A Appendix: The case of a loss function independent of the instruments}

Here we consider the extension of our results to the special case in which $C_{i}=0$, so that the policymaker's objective function is independent of the path of the instruments $i_{t}$. In this case, $L_{i i}=0$, so that the matrix is necessarily not invertible, as assumed in the text. In this case, we cannot solve (3.3) for $i_{t}$. However, in this case we also must have $L_{i y}=0$; hence there is no need to solve (3.3) in order to eliminate $i_{t}$ from (3.2), as the equation is already in the desired form. We can simply take the conditional expectation of (3.2) with respect to $I_{t}$ and obtain

$$
A^{\prime} \varphi_{t+1 \mid t}=-L_{y y} y_{t \mid t}+\delta^{-1} \tilde{I}^{\prime} \varphi_{t \mid t}
$$

which is of the same form as the upper half of the system of equations (3.6). The sticking point is that we are unable to eliminate $i_{t}$ from $(2.1)$, as is also necessary in order to derive the system (3.6) in the text.

It is nonetheless possible to derive a system of difference equations of the same general form. We note that in the present case, (3.3) reduces to

$$
B^{\prime} \varphi_{t+1 \mid t}=0
$$

This is a set of restrictions of the same form as those in the system (3.6), and independent of (3.2). Let us suppose that $B$ is of full rank; ${ }^{13}$ then (A.2) is a system of $n_{i}$ linearly independent restrictions, where $n_{i}$ is the number of instruments. Let $g$ be an $\left(n_{X}+n_{x}\right) \times\left(n_{X}+n_{x}-n_{i}\right)$ matrix, the columns of which all linearly independent of one another, and orthogonal to each of the columns of $B$ (so that $g^{\prime} B=0$ ). Then premultiplying $(2.1)$ by $g^{\prime}$ and taking the conditional expectation with respect to $I_{t}$, we obtain

$$
g^{\prime} \tilde{I} y_{t+1 \mid t}=g^{\prime} A y_{t \mid t}
$$

This provides an additional $n_{X}+n_{x}-n_{i}$ linearly independent restrictions, and equations (A.1), (A.2) and (A.3) jointly comprise a system of the form (3.6). In the generic case, the matrix on the left-hand side will be invertible as assumed in the text, and one will obtain a system of the form (3.7).

\footnotetext{
13 This is purely a notational convenience, as our method here can be applied even when it is not. One simply must eliminate redundant equations from (A.2), and augment the number of columns in the matrix $g$ accordingly.
} 


\section{References}

[1] Aoki, Kosuke, (2000a), "On the Optimal Monetary Policy Response to Noisy Indicators," working paper, Kobe University.

[2] Aoki, Kosuke (2000b), "An Optimal Commitment Plan under Incomplete Information," working paper, Kobe University.

[3] Chow, Gregory C. (1975), Analysis and Control of Dynamic Economic Systems, John Wiley \& Sons, New York.

[4] Dotsey, Michael, and Andreas Hornstein (2000), "Is the Behavior of Money Useful to a Discretionary Policymaker?" working paper, Federal Reserve Bank of Richmond.

[5] Kalchbrenner, J.H., and Peter A. Tinsley (1975), "On the Use of Optimal Control in the Design of Monetary Policy," Special Studies Paper No. 76, Federal Reserve Board.

[6] LeRoy, Stephen F., and Roger N. Waud (1977), "Applications of the Kalman Filter in Short-run Monetary Control," International Economic Review 18, 195-207.

[7] Lucas, Robert E., Jr. (1973), "Some International Evidence on Output-Inflation Tradeoffs," American Economic Review 63, 326-334.

[8] Orphanides, Athanasios (1997), "Monetary Policy Rules Based on Real-Time Data," Finance and Economics Discussion Series No. 1998-03, Federal Reserve Board.

[9] Pearlman, Joseph G. (1986), "Diverse Information and Rational Expectations Models," Journal of Economic Dynamics and Control 10, 333-338.

[10] Sargent, Thomas J. (1991), "Equilibrium with Signal Extraction from Endogenous Variables," Journal of Economic Dynamics and Control 15, 245-273.

[11] Svensson, Lars E.O. (1999), "Inflation Targeting as a Monetary Policy Rule," Journal of Monetary Economics 43, 607-654.

[12] Svensson, Lars E.O., and Michael Woodford (2000), "Indicator Variables for Optimal Policy," NBER Working Paper No. 7953.

[13] Svensson, Lars E.O., and Michael Woodford (2001), "Optimal Policy with Partial Information in a Forward-Looking Model: Certainty-Equivalence Redux," in preparation. 
[14] Townsend, Robert M. (1983), "Forecasting the Forecasts of Others," Journal of Political Economy 91, 546-588.

[15] Woodford, Michael (1999), "Optimal Monetary Policy Inertia," NBER Working Paper No. 7261.

[16] Woodford, Michael (2000a), "Pitfalls of Forward-Looking Monetary Policy," American Economic Review: Papers and Proceedings 90, 100-104.

[17] Woodford, Michael (2000b), "A Neo-Wicksellian Framework for the Analysis of Monetary Policy," working paper, Princeton University.

[18] Woodford, Michael (2001), "Inflation Stabilization and Welfare," NBER Working Paper No. 8071 . 